ThE Astrophysical Journal, 482:541-545, 1997 June 10

(C) 1997. The American Astronomical Society. All rights reserved. Printed in U.S.A.

\title{
SOLAR VARIABILITY AND THE RELATION OF FACULAR TO SUNSPOT AREAS DURING SOLAR CYCLE 22
}

\author{
G. A. Chapman, A. M. Cookson, And J. J. Dobias \\ San Fernando Observatory, Department of Physics and Astronomy, California State University, Northridge, Northridge, CA 91330 \\ Received 1996 October 4; accepted 1997 January 2
}

\begin{abstract}
The total irradiance of the Sun has been found to vary mostly because of changes in the areas of dark sunspots and bright faculae. Improved observations, such as those discussed in this paper, are needed to understand better the interplay between these two competing features. In this paper, faculae are determined by observations using a filter centered at the Ca II K line $(393.4 \mathrm{~nm})$ with a bandpass of $0.9 \mathrm{~nm}$. This filter allows the detection of faculae across the entire solar disk rather than just at the limb, as is the case for white-light faculae. Sunspots are detected with a filter at $672.3 \mathrm{~nm}$ with a bandpass of 9.7 $\mathrm{nm}$. The mean ratio of facular to sunspot area was found to be $16.7 \pm 0.51$ for a $7 \frac{1}{2}$ year period during solar cycle 22 but showed a significant increase as the solar cycle progressed. This ratio suggests that the irradiance excess associated with faculae outweighs the irradiance deficit associated with sunspots by about $50 \%$. The facular area also exhibited a quadratic dependence on sunspot area, as suggested by Foukal, but there is no clear evidence of a turnover in facular area at large sunspot areas. Lagged crosscorrelations between facular and sunspot areas showed a clear rotational modulation extending to lags of five to six rotations when spots led faculae. Lags in the opposite direction, however, showed the rotational modulation falling abruptly after about two rotations.
\end{abstract}

Subject headings: Sun: activity — Sun: faculae, plages — sunspots

\section{INTRODUCTION}

The relation between the areas of bright faculae and those of dark sunspots is a fundamental and long-studied quantity in solar physics (Allen 1973). Greater knowledge of this relationship can lead to a better understanding of the causes of variations in solar irradiance. The total solar irradiance, determined from bolometric measurements in space, has been shown to vary over the 11 year sunspot cycle with an amplitude of approximately $0.1 \%$ (Hickey et al. 1989; Fröhlich 1994; Fröhlich et al. 1991; Willson 1994; Willson \& Hudson 1991). However, the smoothed irradiance reaches its peak at times of greatest sunspot number, suggesting that the faculae-sunspot relation is more complicated than a simple linear dependency. Modeling irradiance variations based on sunspot areas (deficits) and various ground-based proxies for bright magnetic features (excesses) has suggested that faculae alone might not explain all the excess irradiance. The magnetic flux, seen as a widely scattered network, may account for much of the excess seen during times of solar maximum (Foukal \& Lean 1988; Foukal, Harvey, \& Hill 1991; Otte 1995). A definitive answer will require a more accurate understanding of the facular to sunspot connection.

\section{OBSERVATIONS}

In this study, ground-based photometric images were used to determine both sunspot and facular areas, and a series of analyses was undertaken in an attempt to quantify their relationship. Both sunspot and facular areas are corrected hemispherical areas. The data used were derived from a $7 \frac{1}{2}$ year record of digital images obtained with the Cartesian Full Disk Telescope No. 1 (CFDT1) at the San Fernando Observatory (SFO). The images, which include the Sun and surrounding sky, contain $512 \times 512$ square pixels $5^{\prime \prime}$ on a side and allow for objective measurements of facular and sunspot areas. Daily observations began with a red filter $(\lambda=672.3 \mathrm{~nm})$ on 1985 March 2. Beginning in 1988 May, additional images were obtained in the Ca II K line $(\lambda=393.4 \mathrm{~nm})$, and beginning in 1990 a third color $(\lambda=472.3 \mathrm{~nm})$ was added. Only the red and $\mathrm{K}$ line images are of importance here.

Sunspot properties are determined from red images taken through a $672.3 \mathrm{~nm}$ filter with a $9.7 \mathrm{~nm}$ bandpass. These images are photometrically calibrated by correcting for the gain and zero-point difference of each photodiode, as well as for other systematic effects (Chapman, Cookson, \& Dobias 1996). Images are then flattened by removing limb darkening, leaving a contrast image. Sunspot pixels are identified from the flattened images as those having a negative contrast equal to or more negative than $-8.5 \%$, a value found to represent quite reliably the outer edge of a sunspot (Brandt, Schmidt, \& Steinegger 1990; Steinegger et al. 1990; Beck \& Chapman 1993). Sunspot areas from the CFDT1 correlate well with those published in the Solar Geophysical Data Bulletin with a correlation coefficient near unity. Facular properties are determined from $\mathrm{Ca}$ II $\mathrm{K}$ line images taken through a $393.4 \mathrm{~nm}$ filter with a $0.9 \mathrm{~nm}$ bandpass. Facular pixels are identified from calibrated and flattened $\mathrm{K}$-line images as those with a positive contrast equal to or greater than a contrast criterion whose lower bound is $4.8 \%$ at disk center and that increases with distance from the center of the disk. This criterion is approximately 2.5-3 standard deviations above the quiet-Sun noise determined in a box at disk center (Chapman et al. 1992; Chapman, Cookson, \& Dobias 1994). All the SFO data presented here were processed on an HP 700-series computer using recently updated, more robust software (Walton et al. 1997).

\section{ANALYSIS}

We examined the ratio of facular to sunspot areas by grouping them in several ways. Initially, we considered the ratio of the entire data set, which spanned an interval of 2771 days from 1988 May 30 through 1995 December 31. 
The result was a mean ratio of $16.7 \pm 0.51$, where the uncertainty is the standard deviation of the mean. This ratio of facular to sunspot area is roughly a factor of 5 larger than that given in Allen (1973), a result not unexpected since the facular areas discussed here were determined from K-line images rather than white-light images. White-light faculae seen near the limb are very similar in shape and area to faculae seen through wide-band K-line filters (Wilson 1981), but they are not visible near the center of the solar disk. However, near disk center faculae can be seen through a $\mathrm{K}$-line filter. Since faculae detected with a wide K-line filter more accurately represent the total area of nonspot magnetic fields, we feel that the facular areas discussed here are the appropriate ones to use when determining the facular to sunspot area ratio. One should keep in mind, however, that this ratio may also depend on both the spatial resolution of the telescope and the bandwidth of the K-line filter. The ratio of facular to sunspot area can be used to estimate the effect on solar luminosity variation through the use of irradiance models for sunspots and faculae (Chapman, Herzog, $\&$ Lawrence 1986). From the coefficients found in Chapman et al. (1996), the effects of faculae balance those due to sunspots when there is a ratio of facular to sunspot area of approximately 11 . Since the ratio of 16.5 found here exceeds that by $50 \%$, the luminosity excess due to faculae outweighs the luminosity deficit due to sunspots by that amount.

The daily facular to sunspot ratios show an increase over the $7 \frac{1}{2}$ year period. To determine the properties of the trend, we binned the data by both calendar year and 100 day bins. This is a low-pass filtering process by which successively increasing bandwidths correspond to decreasing bin lengths. The calendar year grouping produced an annual mean ratio of facular to spot area that showed a statistically significant increase with time. The ratio has a slope of
$1.82 \pm 0.48$ per year over the $7 \frac{1}{2}$ years and a linear correlation coefficient, $r$, of 0.84 . For eight data points, this value of $r$ has a significance level of $p<0.01$. The mean ratio of the 100 day bins showed a somewhat similar and still statistically significant increase with time. Here the slope of the regression was $0.39 \pm 0.12$ per 100 day bin with a correlation coefficient, $r$, of 0.53 for 28 points, which has a significance level of $p<0.004$. Each point was weighted by the inverse of the error. The errors are dominated by random solar variations. For a calendar year grouping, this slope corresponds to approximately 1.96 per year. A linear least squares fit to the time series for the 100 day binning is given here:

$$
\frac{A_{f}}{A_{s}}=13.2 \pm 1.5+(0.39 \pm 0.12) \times \text { bin no. }
$$

where $A_{f}$ is facular area and $A_{s}$ is sunspot area. Figure 1 shows the data used to obtain the coefficients, as well as the line represented by equation (1). The run of the data points in Figure 1 resembles that shown by Lawrence (1987a, 1987b). Using unweighted data, the slope of the fit was $0.56 \pm 0.15$ per 100 day bin with $r=0.60$, which has a significance level of $p<0.002$ for 28 data points.

One might argue that the increase in the area ratio during the solar cycle is due to the decreasing average area of sunspots over time. To investigate this, all days with zero sunspot area were removed, and the data were again rebinned into 100 day groups. This data set, consisting of 14 points, had a slope of $0.68 \pm 0.20$ per 100 day bin, $r=0.71$, and a significance level of $p<0.005$. Although removing days of zero sunspot area increase the slope (0.56-0.68), the statistical significance of this ratio of facular to sunspot area is somewhat lower.

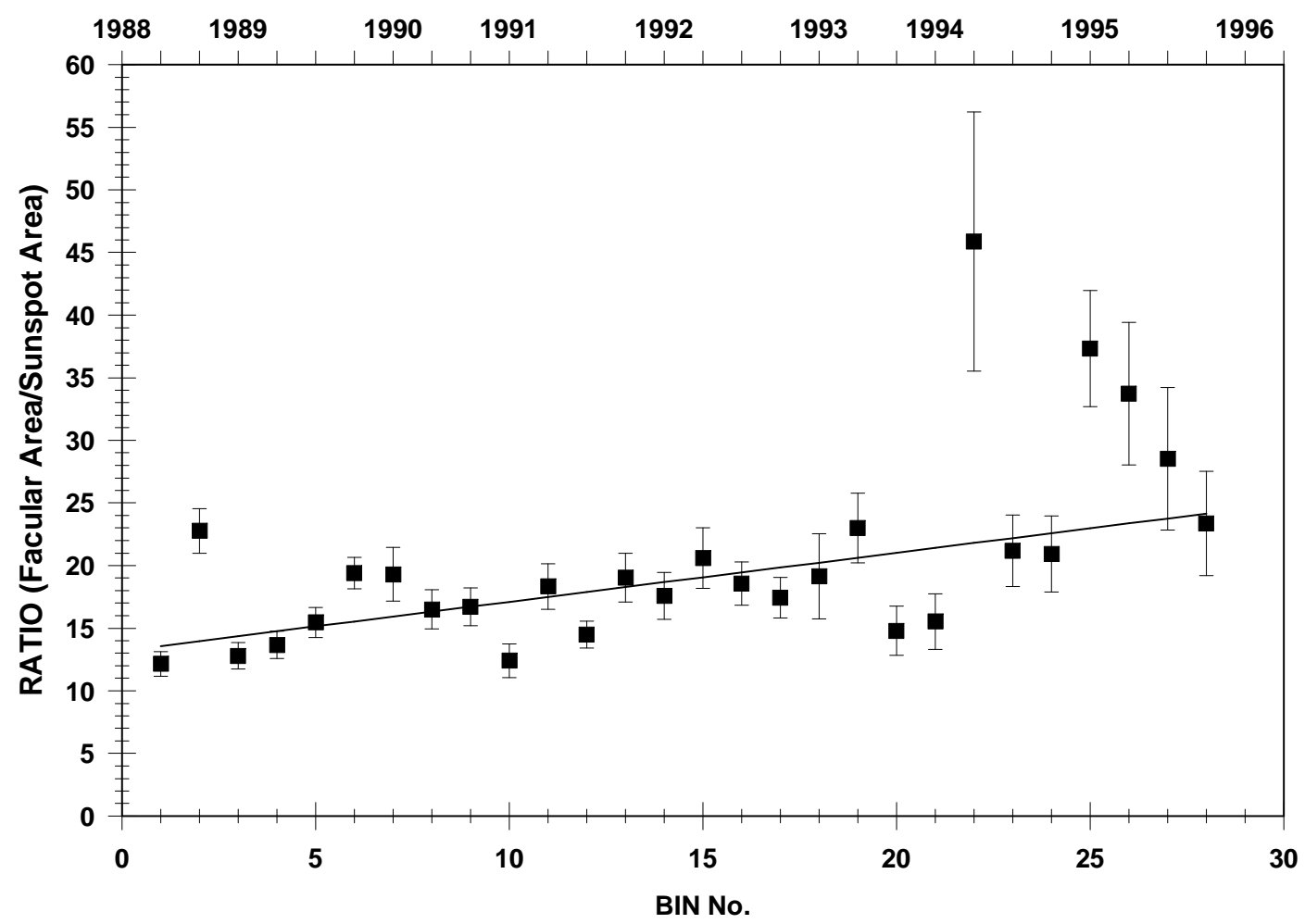

FIG. 1.-Ratios of facular to sunspot area averaged in 100 day bins. The straight line is the regression line given in eq. (1) from a weighted fit. Error bars are standard deviations, which are dominated by random solar fluctuations. 
We examined the relation between facular and sunspot area by using regressions of facular area on sunspot area. Again, the data were grouped into 365 day and 100 day bins. In both cases, regressions showed highly significant correlations for both linear and quadratic representations. Regressions for annual means gave $r=0.84(p<0.01)$ for the linear expression and $r=0.95(p<0.001)$ for the quadratic expression, where the coefficient for the quadratic term is positive. Regressions for the 100 day bins gave $r=0.95$ for the linear dependence and $r=0.97$ for a quadratic dependence but with a negative quadratic coefficient. Partial regression coefficients show that the linear term in the regression expression explains approximately $75 \%$ of the variance, whereas the quadratic term explains only about $36 \%$ of the variance. We conclude that although the quadratic term in equation (2) is statistically significant, facular area does not decrease with increasing sunspot area, as found by Foukal $(1993,1994)$. The explanation for our result may be due in part to our use of K-line faculae seen across the entire solar disk, whereas Foukal relied on whitelight measurements of limb faculae from the Royal Greenwich Observatory (RGO). It may also be partly due to our smaller database compared to that of the RGO.

The results of a weighted regression for the quadratic fitted to the 100 day binned data are given in equation (2), where the weights are inversely proportional to the standard errors in the vertical axis:

$$
\begin{aligned}
A_{f}=414 \pm 725+ & (21 \pm 3) \\
& \times A_{s}-\left[(3.6 \pm 1.4) \times 10^{-3}\right] \times A_{s}^{2} .
\end{aligned}
$$

The parameters in equation (2) are approximately an order of magnitude or more greater than those found by Foukal $(1993,1994)$ using white-light faculae data from the RGO. As mentioned before, this should not be surprising since white-light faculae are very difficult to see at disk positions inside 0.8 of the solar radius. While the measurement of sunspot areas is relatively straightforward, the measurement of facular areas is much more difficult, especially since the definition of faculae is dependent on the wavelength and bandwidth of the observations. Figure 2 shows the data and the least-squares fit of equation (2).

A third way of examining facular and sunspot area was an analysis of the temporal relation between these areas using both lagged cross- and autocorrelations. First, a lagged cross-correlation was calculated, according to equation (3), over the entire interval, omitting days with missing data when summing:

$$
r_{j}\left(A_{f}, A_{s}\right)=\frac{\sum_{i}\left(A_{f}^{i}-\overline{A_{f}}\right) \times\left(A_{s}^{i+j}-\overline{A_{s}}\right)}{\left[\sum_{i}\left(A_{f}^{i}-\overline{A_{f}}\right)^{2} \times \sum_{i}\left(A_{s}^{i}-\overline{A_{s}}\right)^{2}\right]^{1 / 2}} .
$$

The lags ranged from -324 days to +324 days, where negative lags were those in which faculae trailed sunspots and positive lags were those in which faculae led sunspots. Figure 3 shows this lagged cross-correlation. The zero-lag cross-correlation coefficient is 0.75 for 1602 pairs of data points. A 27 day rotational modulation with decreasing amplitude can be seen extending to a lag of approximately -150 days, or nearly five solar rotations. Between -150 and -180 days the pattern disappears, only to reappear beyond a lag of about -180 days as a 27 day modulation but with an opposite phasing. For positive lags, the amplitude of the rotational modulation falls off much more rapidly than for negative lags, and the modulation itself lasts for only one or two rotations. However, it also seems to reappear after a lag of about 180 days.

Next, an autocorrelation of the facular area for the same 2771 day interval, out to a lag of 324 days, displays the same rotational modulation (Fig. 4). Again, one can see the attenuation of the rotational modulation at lags of approximately 130-170 days. As in the case of the cross-

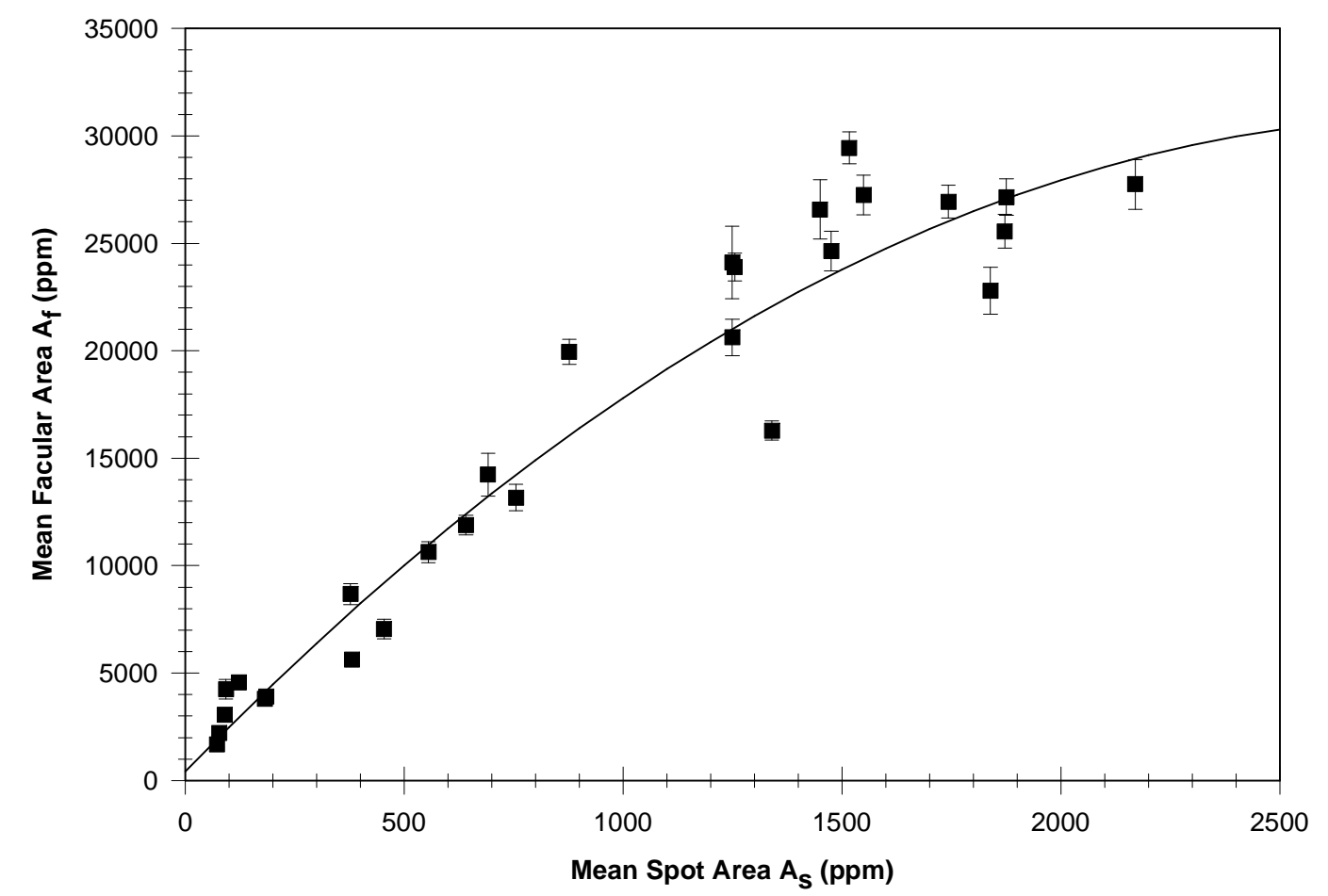

FIG. 2.-Quadratic relation of facular area to sunspot area. The regression line from a weighted fit is that of eq. (2). Error bars are standard deviations, which are dominated by random solar fluctuations. 


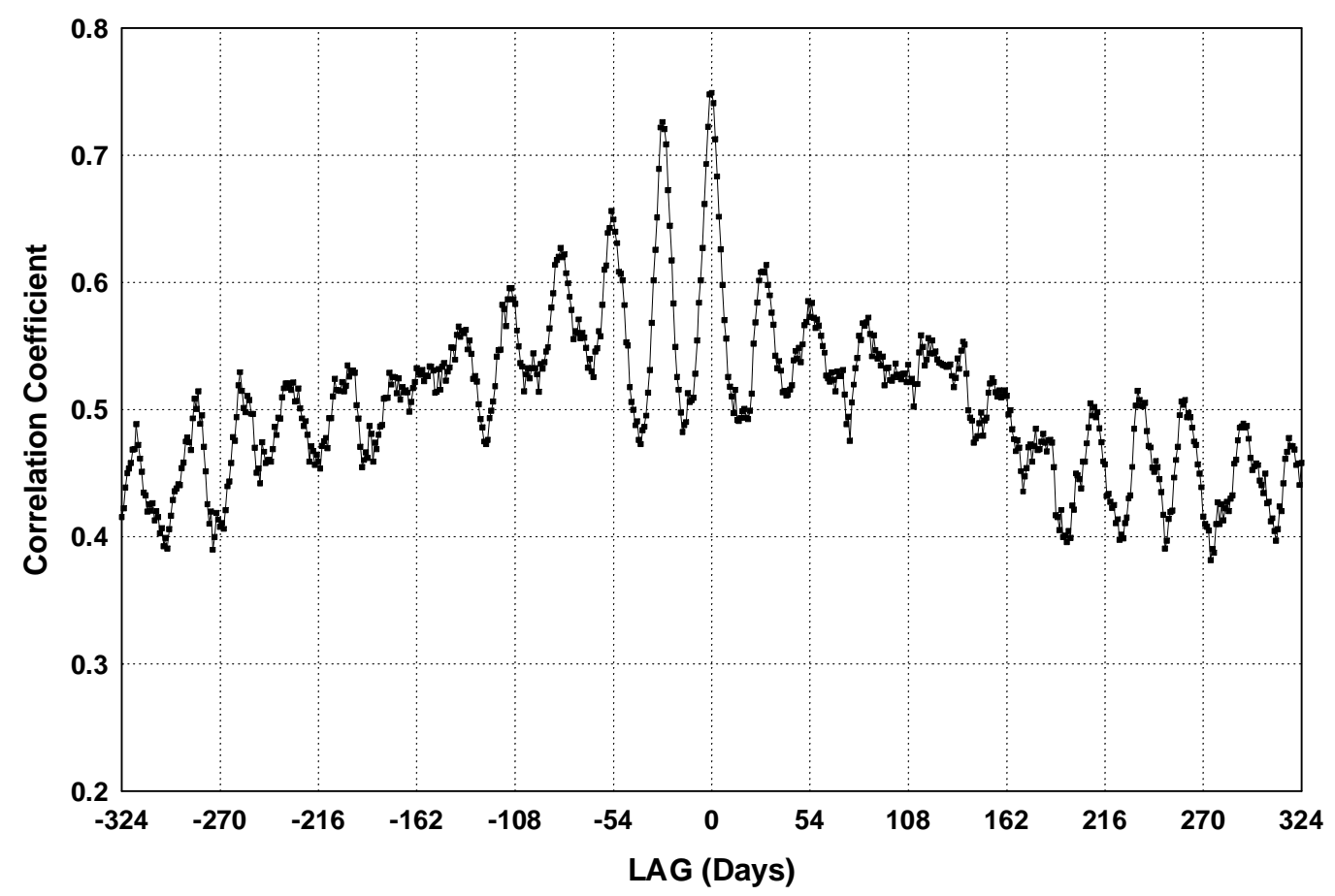

FIG. 3.-Lagged cross-correlation between facular areas and sunspot areas. Days with missing data are not included in the sums. At zero lag there are 1602 pairs of data points.

correlation, the rotational modulation becomes evident again beyond a lag of about 180 days and displays a different phasing.

The rotational modulation is caused by the repeated appearance of solar activity at the same solar longitude. We suggest that the disappearance of the 27 day rotational modulation at lags of about -150 to -160 days in facular to sunspot cross-correlation and the facular autocorrelation corresponds to the typical lifetime of sunspot nests (Trellis
1971; Castenmiller, Zwaan, \& van der Zalm 1986; Brouwer \& Zwaan 1990; van Driel-Gesztelyi, van der Zalm, \& Zwaan 1992) or activity complexes (Bumba \& Howard 1965; Gaizauskas et al. 1983). This time interval also corresponds to that sometimes found for the occurrence of solar flares (Bai 1987). The appearance of the rotational modulation at time intervals greater than 160-180 days with a different phase is probably due to the appearance of an activity complex at a different solar longitude.

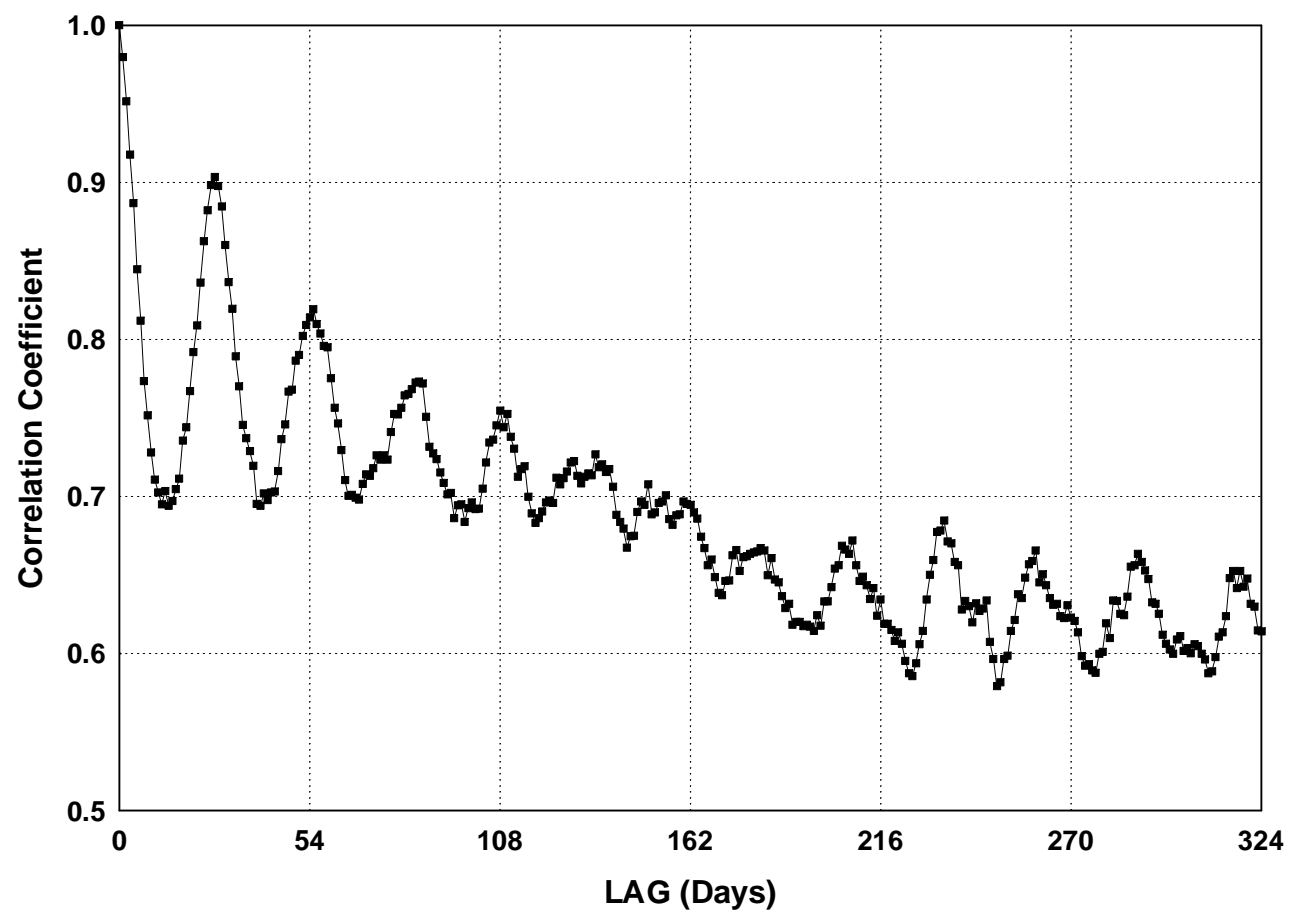

FIG. 4.- Lagged autocorrelation of facular areas. Days with missing data are not included in the sums. 


\section{CONCLUSIONS}

In summary, we found that the ratio of facular to sunspot area appears to be a function of the solar cycle. For a $7 \frac{1}{2}$ year period that includes the onset of significant solar activity during 1988, as well as a decline in activity in 1994 and 1995 , the ratio of facular to sunspot area has a mean value of $16.7 \pm 0.51$. This mean ratio suggests that faculae have a $50 \%$ greater influence on solar luminosity than sunspots. The ratio rises from about 13 in 1988 to about 26 in 19941995. The rise appears to be real and not due to the increasing number of days without sunspots near the end of the cycle, although the increase in the error bars toward the end of this interval makes it difficult to be certain. The magnitude of these ratios may be dependent on spatial resolution and the bandwidth of the K-line filter. Whether the rise in the ratios with phase of the solar cycle is affected by these factors remains to be seen. We confirm the existence of a quadratic relation of facular area to sunspot area and that it differs greatly from a similar relation based on the areas of white-light faculae. From lagged correlations, we find evidence for long-lived eruptions of magnetic activity with lifetimes of approximately $140-160$ days.

We appreciate the efforts of California State University, Northridge, students, especially those serving on the Solar Patrol, who obtained many of these observations. We acknowledge the software support from Stephen Walton that has made the data processing so easy and robust. We appreciate constructive comments by $\mathrm{O}$. $\mathrm{R}$. White on an earlier manuscript that resulted in improvements to this one. This research has been partially supported by NSF grant ATM 95-04374 and NASA grant NAGW-3017.
Allen, C. W. 1973, Astrophysical Quantities (London: Athlone)

Bai, T. 1987, ApJ, 318, L85

Beck, J. G., \& Chapman, G. A. 1993, Sol. Phys., 146, 49

Brandt, P. N., Schmidt, W., \& Steinegger, M. 1990, Sol. Phys., 129, 191

Brouwer, M. P., \& Zwaan, C. 1990, Sol. Phys., 129, 221

Bumba, V., \& Howard, R. 1965, ApJ, 141, 1502

Castenmiller, M. J. M., Zwaan, C., \& van der Zalm, E. B. J. 1986, Sol. Phys., 105, 237

Chapman, G. A., Cookson, A. M., \& Dobias, J. J. 1994, ApJ, 432, 403

. 1996, J. Geophys. Res, 101, 13,541

Chapman, G. A., Herzog, A. D., \& Lawrence, J. K. 1986, Nature, 319, 654

Chapman, G. A., Herzog, A. D., Lawrence, J. K., Walton, S. R., Hudson,

H. S., \& Fisher, B. M. 1992, J. Geophys. Res., 97, 8211

Foukal, P. 1993, Sol. Phys., 148, 219

. 1994, Science, 264, 238

Foukal, P., Harvey, K. L., \& Hill, F. 1991, ApJ, 383, L89

Foukal, P., \& Lean, J. 1988, ApJ, 328, 347

Fröhlich, C. 1994, in The Sun as a Variable Star: Solar and Stellar Irradiance Variations, ed. J. M. Pap, C. Fröhlich, H. S. Hudson, \& S. K. Solanki (Cambridge: Cambridge Univ. Press), 28

Fröhlich, C., Foukal, P. V., Hickey, J. R., Hudson, H. S., \& Willson, R. C. 1991, in The Sun in Time, ed. C. P. Sonnett, M. S. Giampapa, \& M. S. Mathews (Tucson: Univ. Arizona Press), 11

\section{REFERENCES}

Gaizauskas, V., Harvey, K. L., Harvey, J. W., \& Zwaan, C. 1983, ApJ, 265, 1056

Hickey, J. R., Alton, B. M., Kyle, H. L., \& Hoyt, D. V. 1989, Space Sci. Rev., 48,321

Lawrence, J. K. 1987a, J. Geophys. Res., 92, 813

. 1987b, Sol. Phys., 110, 73

Otte, K. 1995, M.S. thesis, California State Univ., Northridge

Steinegger, M., Brandt, P. N., Pap J., \& Schmidt, W. 1990, Ap\&SS, 170, 127

Trellis, M. 1971, Circ. R. Acad. Sci. Paris, B272, 549

van Driel-Gesztelyi, L., van der Zalm, E. B. J., \& Zwaan, C. 1992, in ASP Conf. Ser. 27, The Solar Cycle, ed. K. L. Harvey (San Francisco: ASP), 89

Walton, S. R., Chapman, G. A., Cookson, A. M., Dobias, J. J., \& Preminger, D. 1997, in preparation

Willson, R. C. 1994, in The Sun as a Variable Star: Solar and Stellar Irradiance Variations, ed. J. M. Pap, C. Fröhlich, S. K. Solanki, \& H. S. Hudson (Cambridge: Cambridge Univ. Press), 54

Willson, R. C., \& Hudson, H. S. 1991, Nature, 351, 421

Wilson, P. R. 1981, Sol. Phys., 69, 9 